\title{
Postnatal day 7 ethanol treatment causes persistent reductions in adult mouse brain volume and cortical neurons with sex specific effects on neurogenesis
}

\author{
Leon G. Coleman Jr., Ph.D. ${ }^{1}$, Ipek Oguz, Ph.D. ${ }^{1,2}$, Joohwi Lee ${ }^{3}$, Martin Styner, Ph.D. ${ }^{2,3}$, and \\ Fulton T. Crews, Ph.D. ${ }^{1,2}$ \\ ${ }^{1}$ Bowles Center for Alcohol Studies, University of North Carolina at Chapel Hill CB\# 7178, Chapel \\ Hill, NC 27599-7178, United States. \\ 2Department of Psychiatry, University of North Carolina at Chapel Hill, Chapel Hill, NC 27599, \\ United States. \\ ${ }^{3}$ Department of Computer Science, University of North Carolina at Chapel Hill, Chapel Hill, NC \\ 27599, United States.
}

\begin{abstract}
Ethanol treatment on postnatal day seven (P7) causes robust brain cell death and is a model of late gestational alcohol exposure (Ikonomidou et al., 2000). To investigate the long-term effects of P7 ethanol treatment on adult brain, mice received either two doses of saline or ethanol on P7 (2.5g/ $\mathrm{kg}$, s.c., 2 hours apart) and were assessed as adults (P82) for brain volume (using postmortem MRI) and cellular architecture (using immunohistochemistry). Adult mice that received P7 ethanol had reduced MRI total brain volume (4\%) with multiple brain regions being reduced in both males and females. Immunohistochemistry indicated reduced frontal cortical parvalbumin immunoreactive (PV+IR) interneurons (18-33\%) and reduced Cux1+IR layer II pyramidal neurons $(15 \%)$ in both sexes. Interestingly, markers of adult hippocampal neurogenesis differed between sexes, with only ethanol treated males showing increased doublecortin and Ki67 expression (52 and $57 \%$ respectively) in the dentate gyrus, consistent with increased neurogenesis compared to controls. These findings suggest that P7 ethanol treatment causes persistent reductions in adult brain volume and frontal cortical neurons in both males and females. Increased adult neurogenesis in males, but not females, is consistent with differential adaptive responses to P7 ethanol toxicity between the sexes. One day of ethanol exposure, e.g. P7, causes persistent adult brain dysmorphology.
\end{abstract}

\section{Keywords}

Alcohol; sex; neurogenesis; MRI; neurotoxicity; postnatal

\footnotetext{
(C) 2012 Elsevier Inc. All rights reserved.

Corresponding Author: Dr. Fulton T. Crews John Andrews Distinguished Professor Professor of Pharmacology and Psychiatry Director, Bowles Center for Alcohol Studies CB\# 7178 University of North Carolina at Chapel Hill School of Medicine Chapel Hill, N.C. 27599-7178 919-966-5678, fax: 919-966-5679 ftcrews@med.unc.edu.

Publisher's Disclaimer: This is a PDF file of an unedited manuscript that has been accepted for publication. As a service to our customers we are providing this early version of the manuscript. The manuscript will undergo copyediting, typesetting, and review of the resulting proof before it is published in its final citable form. Please note that during the production process errors may be discovered which could affect the content, and all legal disclaimers that apply to the journal pertain.
} 


\section{Introduction}

Fetal alcohol spectrum disorders (FASD) is a group of conditions in which in utero ethanol exposure causes persistent cognitive and behavioral disturbances with or without craniofacial abnormalities (Barr and Streissguth, 2001; Mattson et al., 1997). Brain dysfunction can include IQ reductions, hyperactivity, learning deficits and epilepsy and seizures (Bell et al., 2010; Kodituwakku, 2007; Mattson and Riley, 1998). FASD is common, with one study estimating that the prevalence of FASD in the US may be as high as 2-5\% (May et al., 2009). Neuroimaging studies using Magnetic Resonance Imaging (MRI) have reported reductions in total brain volume with certain brain regions including the corpus callosum, hippocampus, cerebellum and caudate being particularly affected (Norman et al., 2009). Since the prevalence of this disorder is high, and the complications can be severe, it is important to better understand the neurostructural deficits associated with FASD. Rodent studies can be used to model certain features of FASD to study the structural effects of ethanol on the developing brain. We have employed an ethanol treatment paradigm in mice during the first postnatal week. This is an age when neurodevelopment is ongoing, but craniofacial development has completed.

The first postnatal week of life in rodents is considered analogous to the third trimester of human pregnancy (Dobbing and Sands, 1979). Several studies have utilized various ethanol exposure paradigms to find that ethanol causes robust neurodegeneration in multiple brain regions of rodents during the first week of postnatal life (Bonthius and West, 1990; Dikranian et al., 2005; Goodlett and Eilers, 1997; Ikonomidou et al., 2000; Olney et al., 2005; Olney et al., 2002a). Ikonomidou et al. found that one day of ethanol treatment on postnatal day 7 (P7) (2.5g/kg s.c., 2 hours apart) caused robust neuronal cell death in multiple brain regions, with the cortical regions showing substantial vulnerability to toxicity at this developmental age (Ikonomidou et al., 2000). The neurotoxic effects of ethanol were thought to be due to the NMDA receptor antagonistic properties of ethanol leading to caspase-3 activation and apoptotic cell death (Ikonomidou et al., 1999; Olney et al., 2002a). Consistent with these observations, we have shown previously that NMDA receptor antagonism on P7 using MK801 results in a persistent loss of parvalbumin interneurons and layer $\mathrm{V}$ pyramidal neurons in the adult (P80) medial prefrontal cortex (mPFC) (Coleman et al 2009). However, the long-term effects of this $P 7$ ethanol treatment paradigm on the adult brain, and whether it causes structural alterations similar to those found in FASD has not been elucidated. In this study we have investigated the long-term effects of P7 ethanol on the adult brain structure using structural MRI and immunohistochemistry.

The use of structural MRI to assess changes in rodent brain structure is a novel application of this historically clinical technique. Recently, structural MRI has been to investigate the acute effects of early gestational ethanol exposure (GD7, GD8, or GD10) on development, demonstrating the utility of this technique to study FASD (Godin et al., 2010; O'LearyMoore et al., 2010; Parnell et al., 2009). O'Leary-Moore et al have also begun to investigate the effects of early gestational ethanol on the adolescent brain (O'Leary-Moore et al., 2011). However, to our knowledge no study has used a MRI in a model of FASD to study the longterm effects on the adult brain. This is the first study to our knowledge that uses structural MRI to assess changes in adults in a mouse model of late gestational ethanol exposure. Since P7 ethanol treatment causes robust neuronal death, we hypothesized that reductions in brain volume would be detected using MRI. We further hypothesized that volume reductions would be associated with neuron losses that could be detected by immunohistochemistry. Since one day of ethanol exposure was found to cause significant cortical damage on P7 (Ikonomidou et al., 2000), and we have previously shown persistent neuron loss in the mPFC following P7 MK801 treatment (Coleman et al., 2009), we chose to focus on changes in the numbers of parvalbumin interneurons and pyramidal neurons in the frontal cortex. In 
this study we have measured layer II pyramidal neurons rather than layer V pyramidal neurons as in our previous study, since layer II pyramidal project contralaterally to contribute to the volume of the corpus callosum (Molyneaux et al., 2009). Since we measured corpus callosum volume with MRI, this enabled a comparison of corpus callosum volume and layer II callosal projection neuron density.

Alcohol is known to have robust effects on hippocampal neurogenesis. We have shown previously in adult male rats, that ethanol exposure reduces both the number and dendritic growth of newborn neurons in the dentate gyrus (He et al., 2005). Following a 4-day ethanol binge in adult male rats, we have found an immediate impairment in neurogenesis, followed by a wave of enhanced neurogenesis that persists for only two weeks after treatment (Nixon and Crews, 2004). Ethanol treatment in rats during the first week of postnatal life has also been shown to have long-lasting effects on hippocampal neurogenesis; however, there are some differences reported in the literature. Miller et al. made observations similar ours in adults, finding increased numbers of dentate gyrus hippocampal neurons at P30-35 after ethanol treatment between P4 and P12 (Miller, 1995). Ieraci and Herrara found that P7 ethanol treatment $(5 \mathrm{mg} / \mathrm{kg})$ in CD1 mice was associated with reduced neurogenesis later in adult life (P147) using BrdU and doublecortin staining dorsally ( -1.22 to $-1.84 \mathrm{~mm}$ from bregma) but ventrally ( -3.52 to $-3.88 \mathrm{~mm}$ from bregma) (Ieraci and Herrera, 2007).

Klintsova et al. found no differences in hippocampal cytogenesis in male rats at P50 and less survival of new neurons using BrdU labeling at P80 after ethanol treatment from P5-P9 (Klintsova et al., 2007), while Helfer et al. did not see any differences in the density of BrdU + neurons in the dentate gyrus at P72 in rats following prenatal ethanol treatment $(5.25 \mathrm{~g} / \mathrm{kg} /$ day, from P4-9) (Helfer et al., 2009). Therefore, the long-term effect of ethanol exposure during the first postnatal on adult neurogenesis is unclear and may vary across rodent species. We studied the effect of P7 ethanol treatment on hippocampal dentate neurogenesis in C57BL/6 mice at P80 using Ki67 and doublecortin (DCX) in a more central region of the dentate gyrus that was not investigated by Ieraci and Herrera ( -2.3 to -3.08 from bregma). We also investigated both male and female mice to determine whether there are genderspecific effects.

We found that P7 ethanol treatment caused a persistent reduction in adult total brain volume using MRI. Volume reductions were found in almost all brain regions measured. Volume reductions in the cortex and corpus callosum were associated with reductions in frontal cortical parvalbumin interneurons and layer II medial prefrontal pyramidal neurons (callosal projection neurons) measured using immunohistochemistry. Surprisingly, adult males that received P7 ethanol showed increased adult hippocampal neurogenesis, evidenced by increased doublecortin immunoreactivity and Ki67 immunolabeled cells in the dentate gyrus. These findings reveal that P7 ethanol treatment results in structural deficits that persist into adulthood. This suggests that human maternal ethanol consumption during the third trimester may have long-term effects on brain volume and cellular architecture.

\section{Methods}

\section{Animal Treatment}

Pregnant C57BL/6 mothers were ordered from Charles River (Raleigh, NC). Offspring were treated as shown in Figure 1. Pups were given two injections of either saline or ethanol $(20 \% \mathrm{w} / \mathrm{v}, 2.5 \mathrm{~g} / \mathrm{kg}$, s.c. at the area of the neck and the back) two hours apart on P7 in order to reproduce the ethanol administration method described previously (Ikonomidou et al., 2000). Both groups of mice were handled identically, and removed from their mother for the same amount of time. This treatment regimen produces blood alcohol concentrations (BACs) that are above $200 \mathrm{mg} / \mathrm{dl}$ (Ikonomidou et al., 2000), and produces pronounced brain cell death on P7 in both mice and rats (Ikonomidou et al., 2000; Olney et al., 2002b). The 
total dose is $5 \mathrm{gm} / \mathrm{kg}$. A total of 26 mice ( 11 control and 15 ethanol) were used for this study. Previously we found brain region specific changes in adult brain volume using a less neurotoxic treatment (adolescent ethanol treatment) with smaller sample sizes (control $=10$, ethanol = 8) (Coleman et al., 2011). Thus, we expected adequate power to detect region specific changes in volume.

Pregnant mice were followed and litters normalized to 10 at birth. Body weights were taken throughout early life. There was no significant main effect of treatment on weight between P7 and P22 ( $\mathrm{F}=2.194, p=0.15$; Supplemental Figure 1). A transient decrease in body weight on P8 was not statistically significant ( $p=0.27, t$-test). Mice were weaned and separated by gender on P21. Mice were anesthetized and sacrificed by perfusion with $4 \%$ paraformaldehyde as adults on P80 for postmortem brain imaging and immunohistochemistry as described previously (Coleman et al., 2009; Qin et al., 2007).

\section{Adult postmortem MRI brain imaging}

Postmortem brains were prepared and underwent magnetic resonance imaging (MRI) as described previously (Coleman et al., 2011). Whole mouse skulls were scanned at the UNC Biomedical Research Imaging Center (BRIC) using a dedicated 9.4T Bruker small animal scanner. Images $(0.12 \mathrm{~mm} \times 0.12 \mathrm{~mm} \times 0.12 \mathrm{~mm})$ were acquired using the following diffusion weighted 3D RARE sequence: $\mathrm{TR}=0.7 \mathrm{~s}, \mathrm{TE}_{\mathrm{eff}}=23.662 \mathrm{~ms}$, Rare Factor $=3$, RARE echo spacing $=11.3067 \mathrm{~ms}$, diffusion gradient time $\delta=6 \mathrm{~ms}$, diffusion gradient separation $\Delta=$ 12.422 , b value $=1000 \mathrm{~s} / \mathrm{mm}^{2}$, matrix size $=200 \times 125 \times 80, F O V=24.0 \mathrm{~mm} \times 15 \mathrm{~mm} \times 9.6 \mathrm{~mm}$. Images were computed from reconstructed diffusion tensor data and were segmented for volumetric analysis. This sequence allowed for the collection of both structural volumetric and diffusion data. In this study only structural volume changes are reported. Brain regions were segmented automatically using a previously established atlas-based method (Lee et al., 2009). The Brookhaven National Library 3-D MRI Digital Atlas Database of the adult C57BL/6 mouse (www.bnl.gov/medical/RCIBI/mouse) was used for the automatic segmentation of this dataset. Blinded investigators subsequently corrected segmentation errors manually.

\section{Immunohistochemistry}

Immunohistochemical analysis was performed as described previously (Coleman et al., 2009; Crews et al., 2004). Briefly, $40 \mu \mathrm{m}$ sections were incubated with $0.6 \% \mathrm{H}_{2} \mathrm{O}_{2}$ for 30 minutes to remove endogenous peroxidase activity. After washes, sections were incubated with one of the following primary antibodies: Parvalbumin (1:1000, overnight, Sigma), Doublecortin (1:400, 48hr, Santa Cruz Biotechnology), Ki67 (1:200, 48hr, Vector Labs), Cux1 (Santa Cruz, sc-13024). Immunoreactive neurons or pixels per area were counted using Bioquant Image Analysis Software as described (Coleman et al., 2009; Crews et al., 2004). For PV, Ki67 and Cux 1 immunopositive cells were quantified using an unbiased stereologic method, as described previously (Crews et al., 2004; Nixon and Crews, 2002; West et al., 1991). Briefly, a one-in-six series of sections was collected, an $x-y$ grid was placed randomly on the section and an optical dissector thickness was used for counting in the z-axis. Data is presented as +IR cells per region of interest area $\left(\mathrm{mm}^{2}\right)$. The boundaries of the frontal cortex including M1 motor, M2 motor and the medial prefrontal cortex were defined using the mouse brain atlas (Franklin and Paxinos, 2001) as described previously (Coleman et al., 2009; Grobin et al., 2003). Briefly, the anterior and posterior boundaries of the frontal cortex were identified using the appearance of the forceps minor corpus callosum (bregma 1.98) and the genu of the corpus callosum (bregma 1.1) respectively. The medial prefrontal cortex included the anterior cingulate, the infralimbic and limbic cortices as described previously (Coleman et al., 2009; Grobin et al., 2003). 
For DCX+IR, pixel density is used since neuroprogenitor processes are included in the quantification. Images were captured by using an Olympus BX50 Microscope and video camera linked to a computer. The microscope, camera, and software were background corrected and normalized to preset light levels to ensure fidelity of data acquisition. Each section of a one-in-six series containing immunopositive cells was video captured. The dentate gyrus (bregma -2.3 to -3.08 ), is circumscribed in each section for region of interest area quantification. The density of immunopositive-pixels was analyzed by taking a pixel count within the circumscribed region. The outlined area [in square millimeter $\left(\mathrm{mm}^{2}\right)$ ] was determined, and staining density was calculated by dividing the pixel count by the overall area (pixels per square millimeter). The region of interest area was calculated to assure equivalent areas were quantified. For all analyses both hemispheres were assessed and averaged for each section. The 4-5\% decrease in overall brain volume found with MRI did not alter region of interest areas or cell density quantification.

\section{MRI brain structural volume statistics}

Student $t$-tests were performed on whole brain and regional volume measurements. For brain regional assessments, $t$-tests followed by a false discovery rate (FDR) analysis were employed to ascertain statistical significance while accounting for type II errors that may be caused by multiple comparisons. The FDR method reports a $q$-value, which is used to calculate the number of false positives expected. The $q$ values were calculated using the $Q$ value module in the $R$ for Windows Software () R Development Core Team 1995-2009. The following formula was then used to determine the chance of detecting false positives: likelihood of a false positive $=q_{\text {target }} \times\left(\#\right.$ of regions with $\left.q_{\text {calculated }} \leq q_{\text {target }}\right) / \#$ of tests. The threshold for significance was set such that the likelihood of detecting a false positive would be less than 0.025 . For this data set, this corresponded to a $q$-value $<0.032$.

\section{Immunohistochemistry statistics}

A Student's $t$-test was employed to determine statistical significance between treatment and control groups. A $p$-value less than 0.05 was considered significant. When assessing parvalbumin immunoreactive neurons in the adult $\mathrm{mPFC}$, the effect of $\mathrm{P} 7$ ethanol treatment at two locations within the mPFC was analyzed using a 2-way ANOVA with the effect of treatment (vehicle or ethanol) and location (defined as distance from bregma) assessed in the analysis.

\section{Results}

\section{P7 Ethanol treatment reduces adult brain volume measured by MRI}

Ethanol treatment on P7 is known to cause cell death in multiple brain regions (Ikonomidou et al., 2000). It is not clear how this impacts adult brain. We treated pups at P7 and assessed adult total brain volume using structural MRI brain images. This ethanol treatment did not have a significant effect on body weight between ages P7 and P22 $\left(\mathrm{F}_{1,40}=2.194, p=0.15\right.$; Supplemental Figure 1). There was a transient decrease in body weight on P8 following treatment that did not reach statistical significance. There were no significant differences in total brain volume between control males and females, being $440 \mathrm{~mm}^{3}$ and $450 \mathrm{~mm}^{3}$, in males and females respectively. However, $\mathrm{P} 7$ alcohol treatment did reduce adult brain volume in both males and females. A comparison of P7 ethanol treated mice including both sexes indicates a reduction of about 5\% in total brain volume due to P7 ethanol treatment, compared to litter-matched controls (Figure 2B). This reduction in total brain volume was seen in both males and females $(4.4 \%, p<0.007$ and $5.95 \%, p<0.0001$ respectively, $\mathrm{N}=11$ control, 15 ethanol). When analyzing the effect of P7 ethanol on total brain volume in adults in conjunction with sex $(2 \times 2$ ANOVA $)$, there was a significant main effect of treatment $\left(F_{1,23}=35.87, p<0.0001\right)$ but there was not a significant main effect of gender $\left(F_{1,23}=1.77\right.$, 
$\mathrm{P}=0.20$ ) on total brain volume. Thus, $\mathrm{P} 7$ ethanol treatment reduces adult whole brain volumes.

To investigate brain region specific effects of P7 ethanol treatment, images were segmented into multiple regions of interest and normalized to the average volume of litter-matched controls. The volumes of most brain regions were reduced except for the olfactory bulb, cerebellum, and inferior colliculi (Table 1). This shows that the volumes of some regions were preserved following the treatment, and that the sample sizes in this study were sufficient to detect region specific changes. Fourteen of the brain regions showed significant volume reductions ranging from $4.09 \%$ to $6.92 \%(* p<0.05, \mathrm{q}<0.03 ; * * p<0.01, \mathrm{q}<0.03$; $* * * p<0.001, \mathrm{q}<0.03 ; p$-values from $t$-test and $\mathrm{q}$ values from FDR). In general, the volumes of most brain regions were reduced proportionally by P7 ethanol, such that the percentage of the total brain volume of each region was unchanged by P7 ethanol treatment (Tables 2 and 3) consistent with nearly global changes in volume due to ethanol treatment. Post-hoc analyses revealed variances in the volumes of some regions between males and females. For instance, seven brain regions showed significant reductions in females, but not males. However, the magnitudes of the volume changes were similar in both sexes (Tables 2 and 3 ). Differences in the sample sizes between males ( $N=5$ control, 6 ethanol) and females (N=7 control, 9 ethanol) likely accounts for these differences, suggesting similar effects on P7 ethanol on adult brain volume in both sexes. Thus, P7 ethanol treatment decreases adult cortical, hippocampal, forebrain, midbrain and other brain regional volumes in both males and females.

\section{P7 Ethanol treatment results in a reduction in the number of parvalbumin positive interneurons and layer II pyramidal neurons in the adult frontal cortex}

In previous studies we found that P7 dizocilpine treatment, an NMDA receptor antagonist that mimics P7 ethanol neurotoxicity (Ikonomidou et al., 2000), resulted in persistent reductions in adult frontal cortical neurons (Coleman et al., 2009). Since P7 ethanol reduces the volume of the adult neocortex, we investigated adult frontal cortical neuron density using immunohistochemistry specific for a subtype of inhibitory cortical interneurons, e.g. parvalbumin immunoreactive (PV+IR) cells, and cortical layer 2 pyramidal cells, e.g. Cux 1 immunoreactive (Cux1+IR) cells.

P7 ethanol treatment results in a persistent reduction in cortical parvalbumin interneurons focusing within the frontal cortex. Immunohistochemical staining showed visible reductions of PV+IR neurons in the frontal cortex (Figure 3A). Parvalbumin interneuron staining was reduced in both M2 frontal motor cortex (Figure 3B, 31\%, ***p<0.001) and the medial prefrontal cortex (Figure 3C, 19\%, 2-way ANOVA $F_{1,42}=7.08$, *p<0.02, main effect of treatment, $\mathrm{N}=9$ control, 15 ethanol).

MRI volume investigation revealed that $\mathrm{P} 7$ ethanol caused a reduction in volume of the corpus callosum in adults (Table 1). Therefore, we examined whether layer II callosal projection neurons were persistently reduced as a result of P7 ethanol treatment. We performed Cux 1 immunohistochemistry to identify layer II pyramidal neurons. There was a visible reduction in Cux1 immunoreactive cells in the medial prefrontal cortex (Figure 4A). Quantification revealed approximately a $15 \%$ reduction in the density of Cux 1 cells (Figure 4B, $p<0.001, \mathrm{~N}=8$ control, 9 ethanol). Both male and female adults showed similar reductions. Interestingly, there were no reductions in Cux1 pyramidal neurons seen in the M2 frontal motor cortex ( $\mathrm{p}=0.98$, data not shown). This suggests that $\mathrm{mPFC}$ layer II pyramidal neurons are vulnerable to ethanol neurotoxicity on P7. 


\section{P7 Ethanol results in increased cell proliferation and neuronal DCX expression in males but not females}

We have found previously that alcohol can alter neurogenesis in adult males causing an initial reduction in neurogenesis followed by a compensatory increase in neurogenesis up to 14 days after the end of alcohol treatment (Nixon and Crews, 2004). Using DCX and Ki67 immunohistochemistry, we investigated whether P7 ethanol altered adult neurogenesis. DCX+IR was visibly increased in adult male mice that received P7 ethanol (Figure 5A). This corresponded to a 52\% increase in DCX-IR pixels $/ \mathrm{mm}^{2}$ (Figure 5B, N=4 control, 6 ethanol). There was no difference in the areas of dentate gyrus measured between groups $(\mathrm{p}=0.22$, not shown). The entire neuroprogenitor contains doublecortin, such that DCX+IR reflects both the number of DCX+IR cells and the dendritic processes. We also performed immunohistochemical staining for Ki67, a marker of cell proliferation. Ki67 immunoreactivity was increased in adult males that received ethanol on P7 (Figure 6A). When quantified, this corresponded to a 57\% increase in Ki67+cells per area (Figure 6B, $\mathrm{N}=4$ control, 6 ethanol). These observations indicate that increased cell proliferation and immature neuronal outgrowth are found in the dentate gyrus of the hippocampus of adult males exposed to ethanol on P7.

Interestingly, postnatal day 7 ethanol treatment did not result in a change in DCX expression in the dentate gyrus of adult females (Figure 7, N=6 control, 6 ethanol). There was no difference in the area of the dentate gyrus measured between both groups (not shown). This is clearly different than our observations in adult males that received ethanol treatment on P7. Ki67 expression was visualized in the dentate gyrus using immunohistochemistry (Figure 8A). Consistent with DCX measurements in adult females, there were no changes in Ki67 expression in adult females regardless of $\mathrm{P} 7$ ethanol treatment ( $\mathrm{N}=6$ control, 6 ethanol). Quantification revealed similar numbers of Ki67+ neurons in both adult females groups (Figure 8B). Though in certain species such as the rat and vole, the estrous cycle and the level of endogenous estrogen have been found to regulate hippocampal neurogenesis, it has been found in the C57BL/6 mouse that neither the estrous cycle nor ovarectomy alter the rate of adult hippocampal neurogenesis (Lagace et al 2007). Our findings are consistent with no changes in basal neurogenesis between males and females in C57BL/6 mice. Thus, the differences in adult hippocampal DCX and Ki67 expression between males and females following P7 ethanol that we have observed are not explained by the estrous cycle or endogenous estrogen, but rather some other gender specific differences in the response to P7 ethanol exposure.

\section{Discussion}

The developing brain is sensitive to ethanol neurotoxicity during the early postnatal period. We report for the first time a reduction in total brain volume detected by MRI in adult mice that received $\mathrm{P} 7$ ethanol. This observation is consistent with human brain imaging studies and demonstrates the translational utility of this technique. The reduction in brain volume was observed in a majority of the brain regions that were investigated (14 out of 18). Since there were no differences in body weight between the two groups by postnatal day 22 , ethanol effects are likely due to specific effects on the brain rather than effects on overall body size. The nearly global reduction in brain volume may be somewhat broader than what is observed in humans. Human imaging studies have found reductions in total brain volume as well as specific significant reductions in the cortex, corpus callosum, caudate, cerebellum and hippocampus in individuals with FASD (for review see Norman et al 2009). The potentially broader effect seen in rodents may be related to differences in the rate of development between humans and rodents. Developmental events occurring on postnatal day 7 in rodents are occurring during the third trimester in humans (Dobbing and Sands, 1979). However, human pregnancy is 266 days versus 28 days (including in utero and first 
postnatal week) in mice. Thus, this study may be a model of drinking during several days of the third trimester of pregnancy. The ability to detect persistent brain volume changes using MRI demonstrates the utility of this technique and its usefulness in comparing human and rodent studies. Further immunohistochemical investigations also found reductions in cortical neurons.

Several studies have found neuron death acutely after the P7 alcohol treatment paradigm (Ikonomidou et al., 2000; Olney et al., 2005; Olney et al., 2002a). We found persistent reductions in cortical parvalbumin interneurons and layer II pyramidal neurons. These findings are consistent with our previous work in which treatment with the NMDA receptor antagonist MK801 on P7 caused persistent reductions in cortical parvalbumin interneurons and layer V pyramidal neurons (Coleman et al., 2009). This suggests that the loss of neurons contributes to the persistent reduction in brain volume measured using MRI. Other cellular elements as well including dendritic arbors, myelin, and glia could potentially be affected in adulthood by $\mathrm{P} 7$ ethanol, and these structural elements should be investigated. Also, region specific effects were observed. We found that layer II cortical projection pyramidal neurons were reduced in the medial prefrontal cortex, but not in the M2 motor region. This suggests that pyramidal neurons in the mPFC are more vulnerable to the long-term effects of late gestational ethanol toxicity. Layer II pyramidal neurons may play a role in the pathophysiology of FASD. Layer II pyramidal neurons comprise the majority of the corticocortical projection neurons, which send axons contralaterally and comprise the corpus callosum (Molyneaux et al., 2009). Human imaging studies in children with FASD have found reductions in the volume of the corpus callosum (Autti-Ramo et al., 2002; Bhatara et al., 2002; Sowell et al., 2001). We also observed a persistent reduction in the volume of the adult corpus callosum following P7 ethanol treatment, consistent these human studies. A loss of layer II pyramidal neurons could be contributing to the observed volume reduction of corpus callosum in FASD. We also found persistent reductions in parvalbumin interneurons in the adult mPFC and the M2 motor region after P7 ethanol treatment. The mPFC is important for cognitive functions including attentional set shifting and cognitive function (Floresco et al., 2008; Stefani and Moghaddam, 2005). Attentional deficit with hyperactivity disorder is the most common co-morbordity associated with FASD (Bhatara et al., 2006;

Chiodo et al., 2010; Fryer et al., 2007; Kodituwakku et al., 2006). These studies indicate that late gestational ethanol causes persistent reductions in adult layer II pyramidal neurons and parvalbumin interneurons, and that the role of these neurons in the pathophysiology of FASD should be investigated.

We also observed changes in adult neurogenesis after P7 ethanol treatment. Treatment with ethanol on P7 caused an increase in neurogenesis in adult (P80) males evidenced by both increased doublecortin staining and Ki67 expression. This observation shows an enhancement of neurogenesis in adult (P80) mice following P7 ethanol treatment that persists for several weeks longer than we have observed previously following binge ethanol treatment in adult rats (Nixon and Crews, 2004). Interestingly, we did not observe an enhancement of neurogenesis in adult female mice. The reason for this difference is not clear. However, it is likely not attributable to the estrous cycle since neurogenesis in the C57BL/6 mouse does not fluctuate with the estrous cycle as it does in other rodents, but is rather due to some other unknown difference between the two sexes in this species (Lagace et al., 2007). There may have been differences in locomotor activity between males and females. Ethanol treatment on P7 has been found to cause hyperactivity in adult mice at P60 (Ieraci and Herrera, 2006) and exercise is known to increase neurogenesis in the dentate gyrus following ethanol treatment (Crews et al., 2004; Helfer et al., 2009). It is possible that the male mice that received ethanol were more active in their cages, resulting in increased hippocampal neurogenesis. Our findings are consistent with those of Miller et al., who found that early postnatal ethanol treatment in rats (between P4 and P12) was associated with 
increased numbers of dentate gyrus hippocampal neurons at P30-35 (Miller, 1995). However, Ieraci and Herrara, found that P7 ethanol treatment $(5 \mathrm{mg} / \mathrm{kg}$ ) in CD1 mice was associated with reduced neurogenesis later in adult life (P147) in dorsal $(-1.22$ to $-1.84 \mathrm{~mm}$ from bregma) but not ventral ( -3.52 to $-3.88 \mathrm{~mm}$ from bregma) dentate gyrus, and found no differences between genders (Ieraci and Herrera, 2007). We used C57BL/6 mice rather than CD1 mice and studied the region of the dentate gyrus between that was more centrally located ( -2.3 to -3.08 from bregma), which might contribute to the differences between our findings. Also, neurogenesis is known to diminish substantially with age (He and Crews, 2007). Thus, it is also possible that the enhancement of neurogenesis that we observed in adult males at P80 diminishes with advanced age, and was therefore not detected by Ieraci and Herrera at P147. Other neurotoxic insults on P7 have been shown to cause increased neurogenesis in adulthood. Dong et al. found increased neurogenesis using BrdU labeling in the $\mathrm{CA} 3$ region of hippocampus of adult female rats (P60) after acute neurotoxicity induced by P7 kainate treatment (Dong et al., 2003). These findings suggest that there may be a compensatory mechanism following early-life hippocampal damage. The exact timing and duration of such a response depends on the nature of the postnatal neurotoxic insult, the rodent species, gender, and the adult age investigated.

In summary, P7 ethanol causes persistent deficits in adult brain structure that can be detected by MRI and immunohistochemistry. These observations are consistent with human imaging studies of FASD. The potential role of persistent losses of pyramidal neurons and parvalbumin interneurons in FASD should be investigated. Also, future studies should investigate strategies to reverse or prevent this long-term ethanol-induced neuropathology.

\section{Supplementary Material}

Refer to Web version on PubMed Central for supplementary material.

\section{Acknowledgments}

The authors wish to thank the National Institute on Alcohol Abuse and Alcoholism (AA06069; AA011605, AA018051, AA20022, AA20023, AA20024), the UNC-Bowles Center for Alcohol Studies and the UNC Neurodevelopmental Disorders Research Center (NINDS R42 NS059095, NIDA P01 DA022446-01, NIMH R41 NS059095) for support.

\section{References}

Autti-Ramo I, Autti T, Korkman M, Kettunen S, Salonen O, Valanne L. MRI findings in children with school problems who had been exposed prenatally to alcohol. Dev. Med. Child Neurol. 2002; 44:98-106. [PubMed: 11848116]

Barr HM, Streissguth AP. Identifying maternal self-reported alcohol use associated with fetal alcohol spectrum disorders. Alcohol. Clin. Exp. Res. 2001; 25:283-287. [PubMed: 11236844]

Bell SH, Stade B, Reynolds JN, Rasmussen C, Andrew G, Hwang PA, Carlen PL. The remarkably high prevalence of epilepsy and seizure history in fetal alcohol spectrum disorders. Alcohol. Clin. Exp. Res. 2010; 34:1084-1089. [PubMed: 20374205]

Bhatara V, Loudenberg R, Ellis R. Association of attention deficit hyperactivity disorder and gestational alcohol exposure: an exploratory study. J. Atten. Disord. 2006; 9:515-522. [PubMed: 16481668]

Bhatara VS, Lovrein F, Kirkeby J, Swayze V 2nd, Unruh E, Johnson V. Brain function in fetal alcohol syndrome assessed by single photon emission computed tomography. S. D. J. Med. 2002; 55:59-62. [PubMed: 11865707]

Bonthius DJ, West JR. Alcohol-induced neuronal loss in developing rats: increased brain damage with binge exposure. Alcohol. Clin. Exp. Res. 1990; 14:107-118. [PubMed: 1689970] 
Chiodo LM, da Costa DE, Hannigan JH, Covington CY, Sokol RJ, Janisse J, Greenwald M, Ager J, Delaney-Black V. The Impact of Maternal Ageon the Effects of Prenatal Alcohol Exposure on Attention. Alcohol. Clin. Exp. Res. 2010

Coleman LG Jr. He J, Lee J, Styner M, Crews FT. Adolescent binge drinking alters adult brain neurotransmitter gene expression, behavior, brain regionalvolumes, and neurochemistry in mice. Alcohol. Clin. Exp. Res. 2011; 35:671-688. [PubMed: 21223304]

Coleman LG Jr. Jarskog LF, Moy SS, Crews FT. Deficits in adult prefrontal cortex neurons and behavior following early post-natal NMDA antagonist treatment. Pharmacol. Biochem. Behav. 2009; 93:322-330. [PubMed: 19409920]

Crews FT, Nixon K, Wilkie ME. Exercise reverses ethanol inhibition of neural stem cell proliferation. Alcohol. 2004; 33:63-71. [PubMed: 15353174]

Dikranian K, Qin YQ, Labruyere J, Nemmers B, Olney JW. Ethanol-induced neuroapoptosis in the developing rodent cerebellum and related brain stemstructures. Brain Res. Dev. Brain Res. 2005; 155:1-13.

Dobbing J, Sands J. Comparative aspects of the brain growth spurt. Early Hum. Dev. 1979; 3:79-83. [PubMed: 118862]

Dong H, Csernansky CA, Goico B, Csernansky JG. Hippocampal neurogenesis follows kainic acidinduced apoptosis in neonatal rats. J. Neurosci. 2003; 23:1742-1749. [PubMed: 12629178]

Floresco SB, Block AE, Tse MT. Inactivation of the medial prefrontal cortex of the rat impairs strategy set-shifting, but not reversal learning, using a novel, automated procedure. Behav. Brain Res. 2008; 190:85-96. [PubMed: 18359099]

Franklin; Paxinos. The Mouse Brain in Stereotaxic Coordinates. 2001.

Fryer SL, McGee CL, Matt GE, Riley EP, Mattson SN. Evaluation of psychopathological conditions in children with heavy prenatal alcohol exposure. Pediatrics. 2007; 119:e733-41. [PubMed: 17332190]

Godin EA, O’Leary-Moore SK, Khan AA, Parnell SE, Ament JJ, Dehart DB, Johnson BW, Allan Johnson G, Styner MA, Sulik KK. Magnetic resonance microscopy defines ethanol-induced brain abnormalities in prenatal mice:effects of acute insult on gestational day 7. Alcohol. Clin. Exp. Res. 2010; 34:98-111. [PubMed: 19860813]

Goodlett CR, Eilers AT. Alcohol-induced Purkinje cell loss with a single binge exposure in neonatal rats: a stereological study of temporal windows of vulnerability. Alcohol. Clin. Exp. Res. 1997; 21:738-744. [PubMed: 9194933]

Grobin AC, Heenan EJ, Lieberman JA, Morrow AL. Perinatal neurosteroid levels influence GABAergic interneuron localization in adult rat prefrontal cortex. J. Neurosci. 2003; 23:18321839. [PubMed: 12629187]

He J, Crews FT. Neurogenesis decreases during brain maturation from adolescence to adulthood. Pharmacol. Biochem. Behav. 2007; 86:327-333. [PubMed: 17169417]

He J, Nixon K, Shetty AK, Crews FT. Chronic alcohol exposure reduces hippocampal neurogenesis and dendritic growth of newborn neurons. Eur. J. Neurosci. 2005; 21:2711-2720. [PubMed: 15926919]

Helfer JL, Goodlett CR, Greenough WT, Klintsova AY. The effects of exercise on adolescent hippocampal neurogenesis in a rat model of binge alcohol exposure during the brain growth spurt. Brain Res. 2009; 1294:1-11. [PubMed: 19647724]

Ieraci A, Herrera DG. Nicotinamide protects against ethanol-induced apoptotic neurodegeneration in the developing mouse brain. PLoS Med. 2006; 3:e101. [PubMed: 16478293]

Ieraci A, Herrera DG. Single alcohol exposure in early life damages hippocampal stem/progenitor cells and reduces adult neurogenesis. Neurobiol Dis. 2007; 26:597-605. [PubMed: 17490887]

Ikonomidou C, Bittigau P, Ishimaru MJ, Wozniak DF, Koch C, Genz K, Price MT, Stefovska V, Horster F, Tenkova T, Dikranian K, Olney JW. Ethanol-induced apoptotic neurodegeneration and fetal alcohol syndrome. Science. 2000; 287:1056-1060. [PubMed: 10669420]

Ikonomidou C, Bosch F, Miksa M, Bittigau P, Vockler J, Dikranian K, Tenkova TI, Stefovska V, Turski L, Olney JW. Blockade of NMDA receptors and apoptotic neurodegeneration in the developing brain. Science. 1999; 283:70-74. [PubMed: 9872743] 
Klintsova AY, Helfer JL, Calizo LH, Dong WK, Goodlett CR, Greenough WT. Persistent impairment of hippocampal neurogenesis in young adult rats following early postnatal alcohol exposure. Alcohol. Clin. Exp. Res. 2007; 31:2073-2082. [PubMed: 17949464]

Kodituwakku P, Coriale G, Fiorentino D, Aragon AS, Kalberg WO, Buckley D, Gossage JP, Ceccanti M, May PA. Neurobehavioral characteristics of children with fetal alcohol spectrum disorders in communities from Italy: Preliminary results. Alcohol. Clin. Exp. Res. 2006; 30:1551-1561. [PubMed: 16930218]

Kodituwakku PW. Defining the behavioral phenotype in children with fetal alcohol spectrum disorders: a review. Neurosci Biobehav Rev. 2007; 31:192-201. [PubMed: 16930704]

Lagace DC, Noonan MA, Eisch AJ. Hippocampal neurogenesis: a matter of survival. Am J Psychiatry. 2007; 164:205. [PubMed: 17267780]

Lee J, Jomier J, Aylward S, Tyszka M, Moy S, Lauder J, Styner M. Evaluation of atlas based mouse brain segmentation. SPIE Medical Imaging 2009 Image Processing. 2009; 7259:137-146.

Mattson SN, Riley EP. A review of the neurobehavioral deficits in children with fetal alcohol syndrome or prenatal exposure to alcohol. Alcohol. Clin. Exp. Res. 1998; 22:279-294. [PubMed: 9581631]

Mattson SN, Riley EP, Gramling L, Delis DC, Jones KL. Heavy prenatal alcohol exposure with or without physical features of fetal alcohol syndrome leads to IQ deficits. J Pediatr. 1997; 131:718721. [PubMed: 9403652]

May PA, Gossage JP, Kalberg WO, Robinson LK, Buckley D, Manning M, Hoyme HE. Prevalence and epidemiologic characteristics of FASD from various research methods with an emphasis on recent in-school studies. Dev Disabil Res Rev. 2009; 15:176-192. [PubMed: 19731384]

Miller MW. Generation of neurons in the rat dentate gyrus and hippocampus: effects of prenatal and postnatal treatment with ethanol. Alcohol. Clin. Exp. Res. 1995; 19:1500-1509. [PubMed: 8749817]

Molyneaux BJ, Arlotta P, Fame RM, MacDonald JL, MacQuarrie KL, Macklis JD. Novel subtypespecific genes identify distinct subpopulations of callosal projection neurons. J. Neurosci. 2009; 29:12343-12354. [PubMed: 19793993]

Nixon K, Crews FT. Binge ethanol exposure decreases neurogenesis in adult rat hippocampus. J Neurochem. 2002; 83:1087-1093. [PubMed: 12437579]

Nixon K, Crews FT. Temporally specific burst in cell proliferation increases hippocampal neurogenesis in protracted abstinence from alcohol. J. Neurosci. 2004; 24:9714-9722. [PubMed: 15509760]

Norman AL, Crocker N, Mattson SN, Riley EP. Neuroimaging and fetal alcohol spectrum disorders. Dev Disabil Res Rev. 2009; 15:209-217. [PubMed: 19731391]

O'Leary-Moore SK, Parnell SE, Godin EA, Dehart DB, Ament JJ, Khan AA, Johnson GA, Styner MA, Sulik KK. Magnetic resonance microscopy-based analyses of the brains of normal and ethanol-exposed fetal mice. Birth Defects Res A Clin Mol Teratol. 2010; 88:953-964. [PubMed: 20842647]

O’Leary-Moore SK, Parnell SE, Lipinski RJ, Sulik KK. Magnetic resonance-based imaging in animal models of fetal alcohol spectrum disorder. Neuropsychol Rev. 2011; 21:167-185. [PubMed: 21445552]

Olney J, Tenkova T, Dikranian K, Qin Y, Labruyere J, Ikonomidou C. Ethanol-induced apoptotic neurodegeneration in the developing C57BL/6 mouse brain. Brain Res, Dev. Brain Res. 2005; 133:115-126.

Olney JW, Tenkova T, Dikranian K, Muglia LJ, Jermakowicz WJ, D’Sa C, Roth KA. Ethanol-induced caspase-3 activation in the in vivo developing mouse brain. Neurobiol. Dis. 2002a; 9:205-219. [PubMed: 11895372]

Olney JW, Tenkova T, Dikranian K, Qin YQ, Labruyere J, Ikonomidou C. Ethanol-induced apoptotic neurodegeneration in the developing C57BL/6 mouse brain. Brain Res. Dev. Brain Res. 2002b; 133:115-126.

Parnell SE, O’Leary-Moore SK, Godin EA, Dehart DB, Johnson BW, Allan Johnson G, Styner MA, Sulik KK. Magnetic resonance microscopy defines ethanol-induced brain abnormalities in prenatal 
mice: effects of acute insult ongestational day 8. Alcohol. Clin. Exp. Res. 2009; 33:1001-1011. [PubMed: 19302087]

Qin L, Wu X, Block ML, Liu Y, Breese GR, Hong JS, Knapp DJ, Crews FT. Systemic LPS causes chronic neuroinflammation and progressive neurodegeneration. Glia. 2007; 55:453-462. [PubMed: 17203472]

Sowell ER, Mattson SN, Thompson PM, Jernigan TL, Riley EP, Toga AW. Mapping callosal morphology and cognitive correlates: effects of heavy prenatal alcohol exposure. Neurology. 2001; 57:235-244. [PubMed: 11468307]

Stefani MR, Moghaddam B. Systemic and prefrontal cortical NMDA receptor blockade differentially affect discrimination learning and set-shift ability in rats. Behav. Neurosci. 2005; 119:420-428. [PubMed: 15839788]

West MJ, Slomianka L, Gundersen HJ. Unbiased stereological estimation of the total number of neurons in thesubdivisions of the rat hippocampus using theoptical fractionator. Anat. Rec. 1991; 231:482-497. [PubMed: 1793176] 
Postnatal Day 7 Ethanol Treatment and Adult Analyses

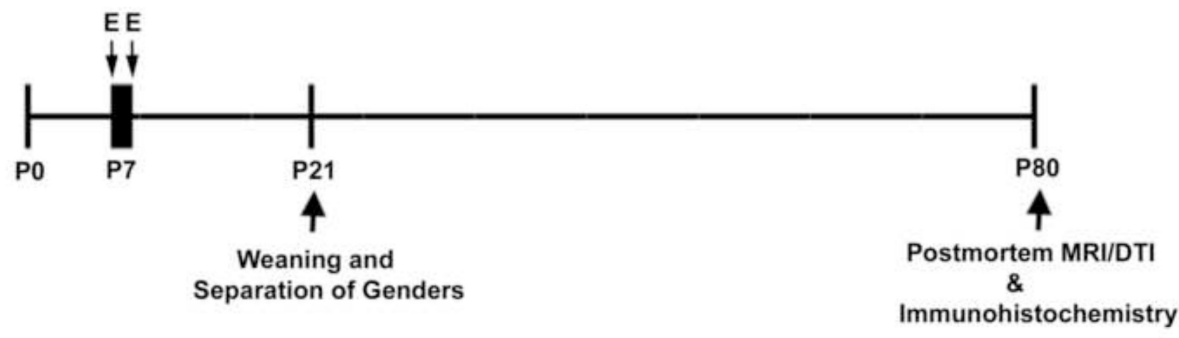

Figure 1.

P7 ethanol treatment with adult (P80) investigation. Mice were treated with 2 ethanol treatments $(2.5 \mathrm{~g} / \mathrm{kg}$,s.c.two hours apart on P7. Postmortem brain imaging and immunohistochemistry were performed in adulthood (P80). Key: E - ethanol. 
A

\section{Adult Brain Volumes}

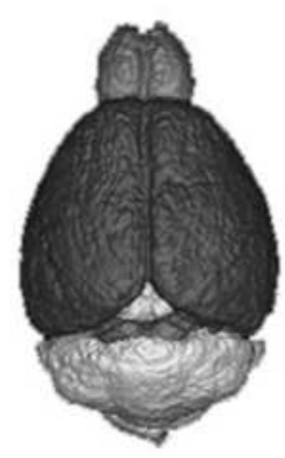

Control

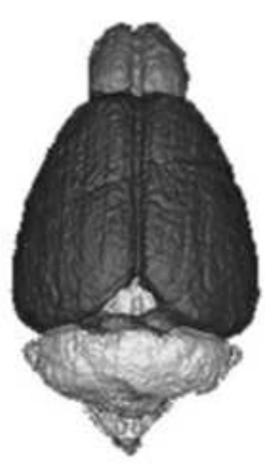

P7 Ethanol
B

Total Brain Volume of All Adults

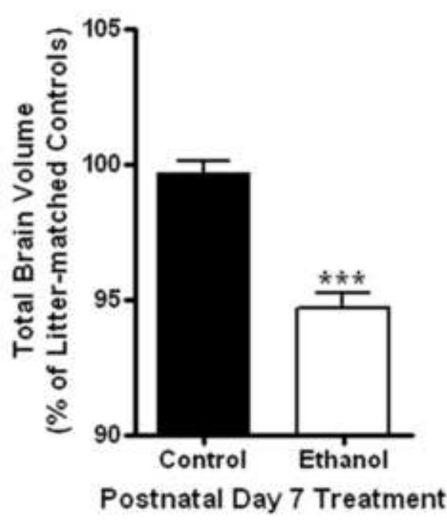

Figure 2.

Reduction in adult total brain volume following P7 ethanol. (A) 3D Renderings of adult subjects that received either saline or ethanol on P7. Note the global reductions in brain volume. (B) Adult brain volumes of all subjects. Adult mice that received P7 ethanol treatment showed approximately a $5 \%$ reduction in total brain volume when compared to litter-matched controls $(* * * \mathrm{p}<0.0001, \mathrm{~N}=11$ control, 15 ethanol) 
A
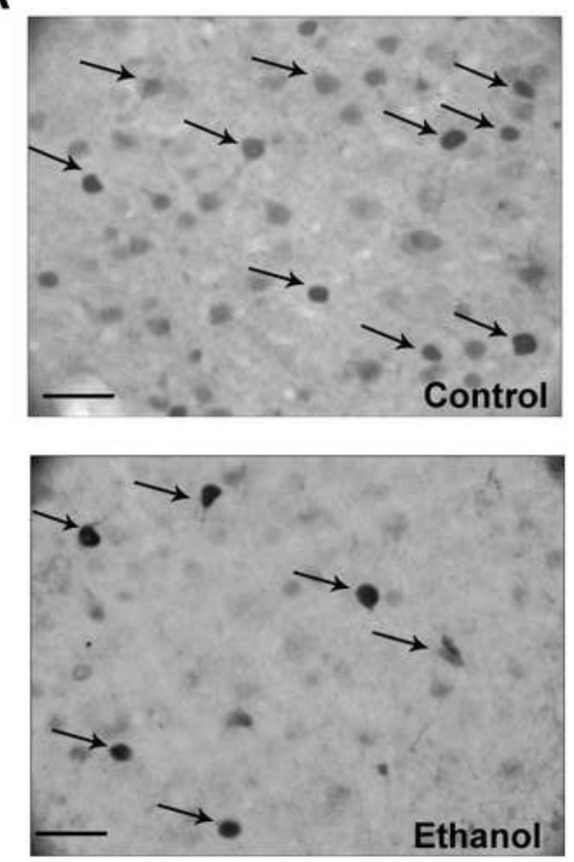

B

Parvalbumin in Frontal Motor Cortex (M2)

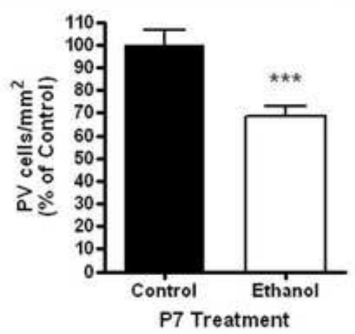

C

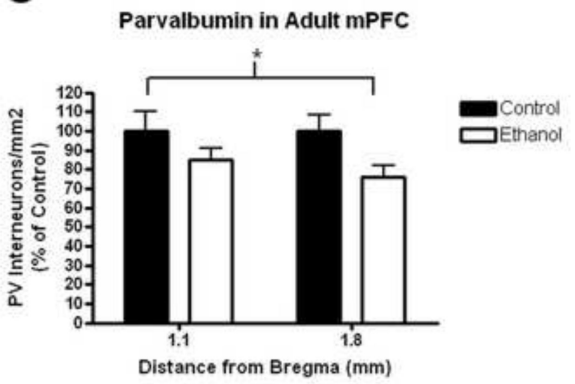

Figure 3.

Reduction of parvalbumin interneurons in the adult frontal cortex following P7 ethanol treatment. Mice were given either saline or ethanol $(2.5 \mathrm{~g} / \mathrm{kg}$, i.p.) two times, two hours apart on P7. Both sexes were pooled. (A) Representative images (40X) of PV+IR staining in the M2 region of the frontal cortex. Visible reductions in PV+IR are seen. (B) Significant reduction in PV+IR neuron density in the frontal M2 motor cortex $(31 \%$, ***p $<0.001)$. (C) Significant reduction of $19 \%$ in PV+IR neuron density across the medial prefrontal cortex from 1.1 to $1.8 \mathrm{~mm}$ distance from bregma (denoted by asterix). 2-way ANOVA, main effect of treatment $F_{1,42}=7.08,{ }^{*} \mathrm{p}<0.02, \mathrm{~N}=9$ control, 15 ethanol). Arrows highlight representative PV+IR neurons. Scale bars denote 20 microns. 
A
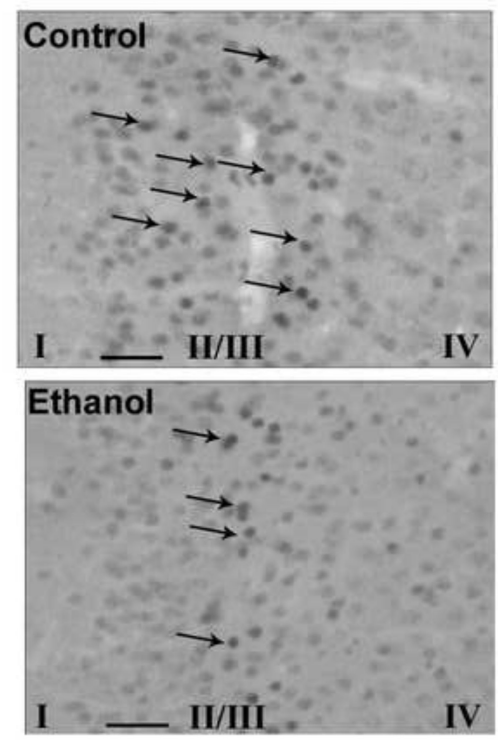

B

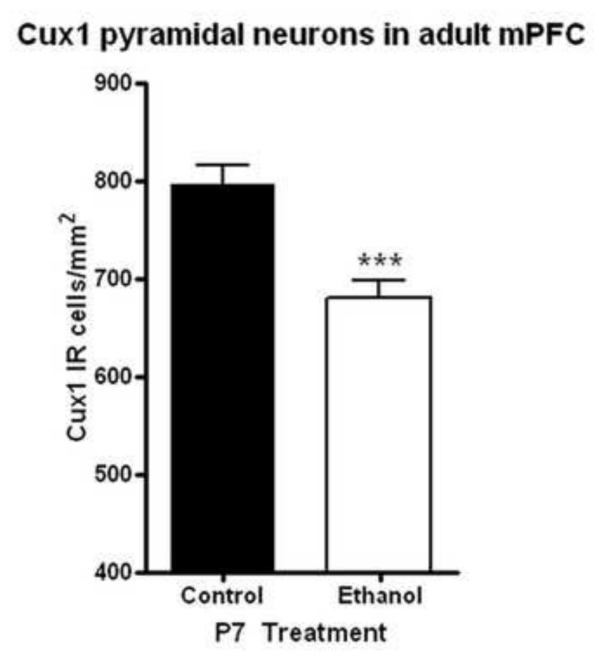

Figure 4.

P7 ethanol reduces Cux1 labeled layer II pyramidal neurons in the medial prefrontal cortex (mPFC). Adult mice that received ethanol $(2.5 \mathrm{~g} / \mathrm{kg}$, i.p. twice, two hours apart) showed fewer Cux1 immunopositive neurons in the mPFC (A) Images (40X) showing fewer Cux1 layer II pyramidal neurons in the mPFC (B) Quantification of Cux1 layer II pyramidal neurons in the mPFC showed approximately a $15 \%$ significant reduction in adult mice that received P7 ethanol $(* * * \mathrm{p}<0.001, \mathrm{~N}=8$ control, 9 ethanol). Arrows highlight representative PV+IR neurons. Scale bars denote 20 microns. Layers I, II/II, and IV are identified using Roman Numerals at the bottom of the images. 


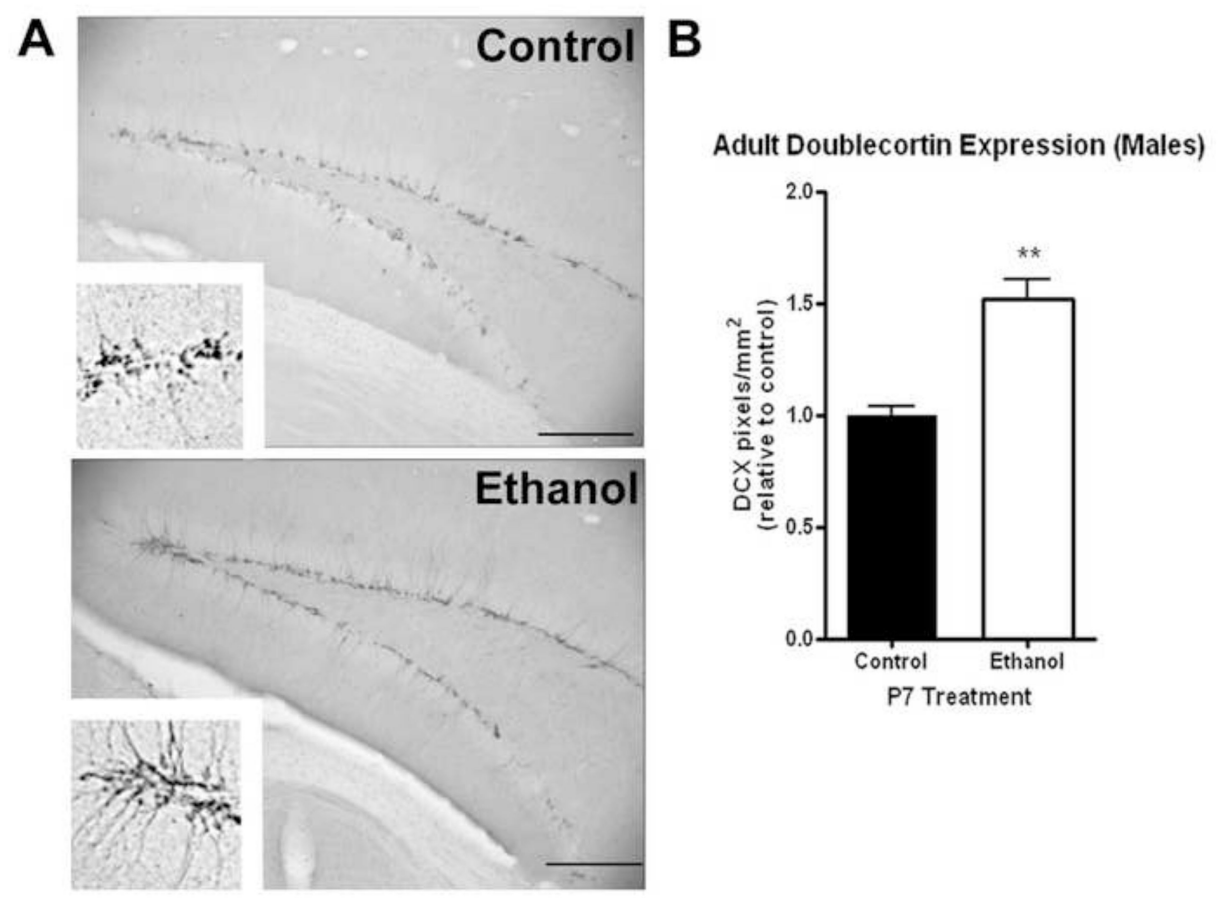

Figure 5.

P7 ethanol alters neurogenesis in adult males. Males that received ethanol $(2.5 \mathrm{~g} / \mathrm{kg}$, i.p. twice, two hours apart) showed an increase doublecortin (DCX) expression in the dentate gyrus. (A) Representative images showing increased DCX expression (Scale bars denote 200 microns). High powered images (120X) are also shown in the inserts to highlight the differences. (B) Quantification of DCX-IR pixels shows a 52\% increase in dentate gyrus DCX expression (**p<0.004). $\mathrm{N}=4$ control, 6 ethanol 
A
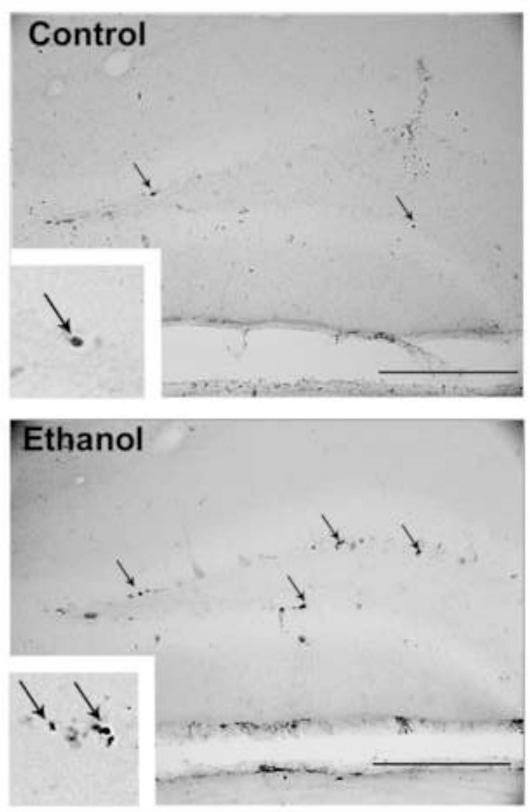

B

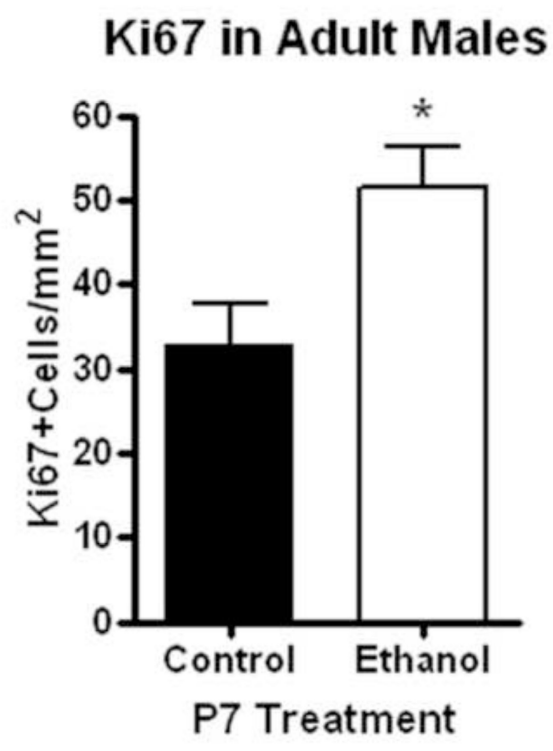

Figure 6.

P7 ethanol alters cell proliferation in adult males. Males that received ethanol $(2.5 \mathrm{~g} / \mathrm{kg}$, i.p. twice, two hours apart) showed an increase Ki67 expression in the dentate gyrus. (A) Representative images showing increased Ki67 expression in the ethanol group. Scale bars denote 100 microns. Arrows highlight Ki67+cells. High powered images (120X) are in the inserts are included. (B) Quantification of Ki67 cells per area showed a 57\% increase in dentate gyrus Ki67 expression (**p<0.004). N=4 control, 6 ethanol. Scale bars denote 100 microns. 
A

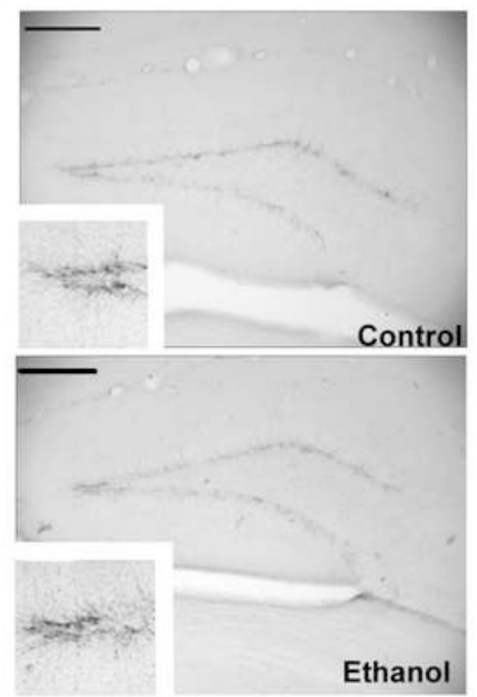

B

\section{Doublecortin Expression (Females)}

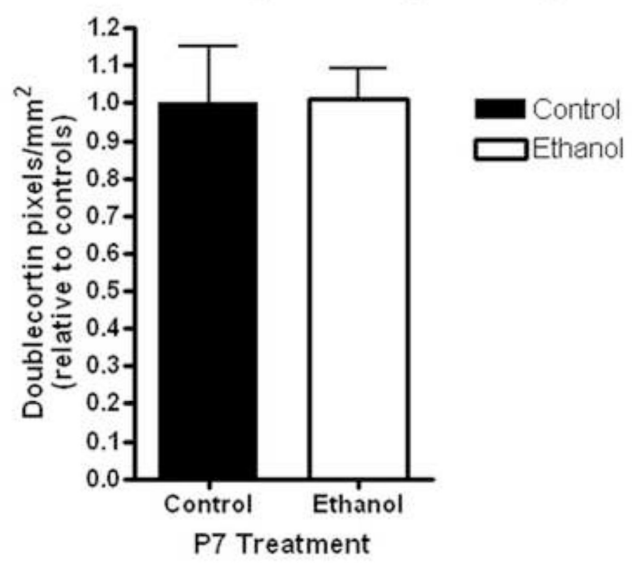

Figure 7.

P7 ethanol does not alter neurogenesis in adult females. Females that received ethanol $(2.5 \mathrm{~g} /$ $\mathrm{kg}$, i.p. twice, two hours apart) showed no change in doublecortin (DCX) expression in the dentate gyrus. (A) Representative images showing similar DCX expression. High powered images (120X) in the inserts also show similar DCX expression. (B) Quantification of DCXIR pixels no change in dentate gyrus DCX expression $(\mathrm{p}<0.95)$. $\mathrm{N}=6$ control, 6 ethanol. Scale bars denote 200 microns. 
A

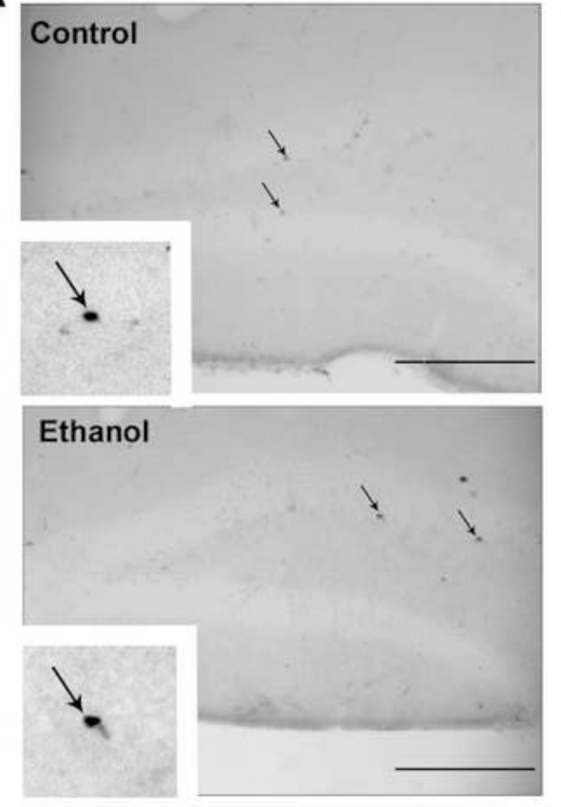

B

\section{Ki67 in Adult Females}

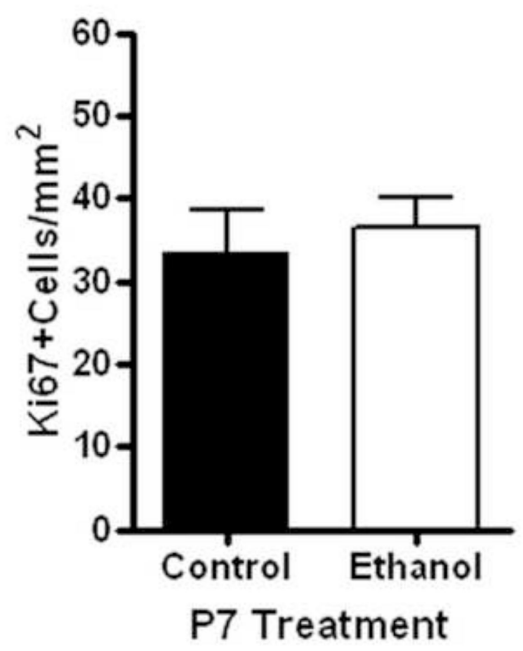

Figure 8.

P7 ethanol does not alter cell proliferation in adult females. Females that received ethanol $(2.5 \mathrm{~g} / \mathrm{kg}$, i.p. twice, two hours apart) showed no change in Ki67 expression in the dentate gyrus. (A) Representative images showing similar Ki67 expression in both groups. Scale bars denote 100 microns. High powered images (120X) are in the inserts and also show no difference. (B) Quantification of Ki67 cells per area showed a similar density of Ki67 cells in both treatment groups. $\mathrm{N}=5$ control, 6 ethanol. 
Table 1

Regional brain volumes assessment (all subjects)

\begin{tabular}{|l|l|l|l|}
\hline Region & \% Difference & $\boldsymbol{p}$-value $(\boldsymbol{t}$-test) & $\begin{array}{l}\text { q-value } \\
\text { (FDR) }\end{array}$ \\
\hline Neocortex & $-6.37^{* * *}$ & 0.0003 & 0.001 \\
\hline Hypothalamus & $-6.92^{* * *}$ & 0.0003 & 0.001 \\
\hline Amygdala & $-6.33^{* * *}$ & 0.0005 & 0.001 \\
\hline Hippocampus & $-4.75^{* *}$ & 0.0010 & 0.001 \\
\hline Caudate \& Putamen & $-4.40^{* *}$ & 0.0010 & 0.001 \\
\hline Basal Forebrain \& Septum & $-6.62^{* *}$ & 0.0011 & 0.001 \\
\hline Thalamus & $-4.28^{* *}$ & 0.0019 & 0.002 \\
\hline Internal Capsule & $-4.28^{* *}$ & 0.0019 & 0.002 \\
\hline Globus Pallidus & $-4.3^{* *}$ & 0.0026 & 0.002 \\
\hline Central Gray & $-4.09^{* *}$ & 0.0035 & 0.002 \\
\hline Rest of Midbrain & $-4.44^{* *}$ & 0.0052 & 0.003 \\
\hline Superior Colliculi & $-4.17^{* *}$ & 0.0074 & 0.004 \\
\hline Corpus Callosum/Ext Capsule & $-4.40^{* *}$ & 0.0078 & 0.004 \\
\hline Fimbria & $-4.82^{*}$ & 0.011 & 0.006 \\
\hline Brain Stem & $-5.95^{*}$ & 0.030 & 0.015 \\
\hline Cerebellum & -3.85 & 0.059 & 0.028 \\
\hline Olfactory Bulb & -4.98 & 0.23 & 0.10 \\
\hline Inferior Colliculi & +0.26 & 0.95 & 0.40 \\
\hline
\end{tabular}

$\mathrm{FDR}=$ False discovery rate correction for multiple comparisons

$p=t$-test probability

$\mathrm{q}=$ FDR value, threshold for significance is $\mathrm{q}<0.03$

$p<\mathrm{lt} ; 0.05$ and $\mathrm{q}<\mathrm{lt} ; 0.03$

$* *$

$p<1 \mathrm{t} ; 0.01$ and $\mathrm{q}<\mathrm{lt} ; 0.03$

$* * *$

$p<1 \mathrm{t} ; 0.001$ and $\mathrm{q}<1 \mathrm{t} ; 0.03$

(-) reduction in volume

(+) increase in volume 
Table 2

Regional brain volumes in males assessed by MRI

\begin{tabular}{|l|r|r|c|c|}
\hline Brain Region & $\begin{array}{r}\text { Control }\left(\mathbf{m m}^{\mathbf{3}}\right) \\
\text { mean } \mathbf{\text { S.D. }} .\end{array}$ & $\begin{array}{r}\text { Ethanol }\left(\mathbf{m m}^{\mathbf{3}} \mathbf{)}\right. \\
\text { Mean } \mathbf{\text { S.D. }}\end{array}$ & $\begin{array}{c}\text { Control } \\
\text { \% TBV }\end{array}$ & $\begin{array}{c}\text { Ethanol } \\
\text { \% TBV }\end{array}$ \\
\hline Hippocampus & $24.3 \pm 0.5$ & $23.6 \pm 0.8$ & 5.5 & 5.6 \\
\hline CC \& External capsule & $12.9 \pm 0.4$ & $12.5 \pm 0.3$ & 2.9 & 2.9 \\
\hline Caudate \& Putamen & $24.8 \pm 0.4$ & $24.3 \pm 1.0$ & 5.6 & 5.7 \\
\hline AC & $1.2 \pm 0.0$ & $1.2 \pm 0.0$ & 0.3 & 0.3 \\
\hline GP & $3.0 \pm 0.1$ & $3.0 \pm 0.1$ & 0.7 & 0.7 \\
\hline Internal capsule & $2.2 \pm 0.1$ & $2.1 \pm 0.1$ & 0.5 & 0.5 \\
\hline Thalamus & $24.7 \pm 0.5$ & $24.3 \pm 1.0$ & 5.6 & 5.7 \\
\hline Cerebellum & $54.0 \pm 1.3$ & $52.3 \pm 2.3$ & 12.2 & 12.3 \\
\hline Superior Colliculi & $7.9 \pm 0.1$ & $7.7 \pm 0.4$ & 1.8 & 1.8 \\
\hline Ventricle & $1.5 \pm 0.1$ & $1.4 \pm 0.1$ & 0.3 & 0.3 \\
\hline Hypothalamus & $11.9 \pm 0.4$ & $11.3 \pm 0.5$ & 2.7 & 2.7 \\
\hline Inferior colliculi & $5.4 \pm 0.0$ & $5.3 \pm 0.3$ & 1.2 & 1.2 \\
\hline Central Gray & $4.2 \pm 0.1$ & $4.2 \pm 0.1$ & 0.9 & 1.0 \\
\hline Neocortex & $144.7 \pm 2.4$ & $140.5 \pm 4.1$ & 32.7 & 33.2 \\
\hline Amygdala & $12.8 \pm 0.5$ & $12.1 \pm 0.3$ & 2.9 & 2.8 \\
\hline Olfactory Bulb & $27.0 \pm 1.5$ & $24.0 \pm 2.8$ & 6.1 & 5.7 \\
\hline Brain Stem & $49.7 \pm 2.1$ & $48.1 \pm 4.0$ & 11.2 & 11.4 \\
\hline Rest of Midbrain & $12.2 \pm 0.2$ & $11.9 \pm 0.5$ & 2.8 & 2.8 \\
\hline Basal Forebrain \& Septum & $12.6 \pm 0.3$ & $12.1 \pm 0.6$ & 2.9 & 2.9 \\
\hline Fimbria & $2.2 \pm 0.1$ & $2.1 \pm 0.1$ & 0.5 & 0.5 \\
\hline
\end{tabular}

$\% \mathrm{TBV}=$ percent of total brain volume

Volumes are presented as mean \pm S.D 
Table 3

Regional brain volumes in females assessed by MRI

\begin{tabular}{|l|r|r|c|c|}
\hline Brain Region & $\begin{array}{r}\text { Control }\left(\mathbf{m m}^{\mathbf{3}}\right) \\
\text { mean } \pm \text { S.D. }\end{array}$ & $\begin{array}{r}\text { Ethanol }\left(\mathbf{m m}^{\mathbf{3}}\right) \\
\text { mean } \pm \text { S.D. }\end{array}$ & $\begin{array}{c}\text { Control } \\
\text { \% TBV }\end{array}$ & $\begin{array}{c}\text { Ethanol } \\
\text { \% TBV }\end{array}$ \\
\hline Hippocampus & $25.4 \pm 0.7$ & $23.8 \pm 1.1$ & 5.6 & 5.6 \\
\hline CC \& External capsule & $13.5 \pm 0.5$ & $12.8 \pm 0.6$ & 3.0 & 3.0 \\
\hline Caudate \& Putamen & $26.1 \pm 0.6$ & $24.6 \pm 1.0$ & 5.8 & 5.8 \\
\hline AC & $1.2 \pm 0.1$ & $1.2 \pm 0.1$ & 0.3 & 0.3 \\
\hline GP & $3.2 \pm 0.1$ & $3.0 \pm 0.1$ & 0.7 & 0.7 \\
\hline Internal capsule & $2.3 \pm 0.1$ & $2.1 \pm 0.1$ & 0.5 & 0.5 \\
\hline Thalamus & $26.0 \pm 0.7$ & $24.4 \pm 1.1$ & 5.8 & 5.7 \\
\hline Cerebellum & $55.3 \pm 4.6$ & $53.3 \pm 3.6$ & 12.3 & 12.5 \\
\hline Superior Colliculi & $8.4 \pm 0.2$ & $8.0 \pm 0.4$ & 1.9 & 1.9 \\
\hline Ventricle & $1.6 \pm 0.1$ & $1.5 \pm 0.1$ & 0.3 & 0.3 \\
\hline Hypothalamus & $12.6 \pm 0.8$ & $11.5 \pm 0.6$ & 2.8 & 2.7 \\
\hline Inferior colliculi & $5.3 \pm 0.8$ & $5.4 \pm 0.3$ & 1.2 & 1.3 \\
\hline Central Gray & $4.4 \pm 0.2$ & $4.2 \pm 0.2$ & 1.0 & 1.0 \\
\hline Neocortex & $147.9 \pm 5.5$ & $135.9 \pm 9.6$ & 32.8 & 31.9 \\
\hline Amygdala & $13.1 \pm 0.6$ & $12.2 \pm 0.7$ & 2.9 & 2.9 \\
\hline Olfactory Bulb & $26.9 \pm 2.5$ & $26.7 \pm 3.3$ & 6.0 & 6.3 \\
\hline Brain Stem & $52.2 \pm 5.0$ & $48.2 \pm 1.4$ & 11.6 & 11.3 \\
\hline Rest of Midbrain & $12.9 \pm 0.4$ & $12.1 \pm 0.6$ & 2.9 & 2.9 \\
\hline Basal Fore Brain \& Septum & $13.1 \pm 0.5$ & $12.1 \pm 0.8$ & 2.9 & 2.8 \\
\hline Fimbria & $2.3 \pm 0.1$ & $2.1 \pm 0.1$ & 0.5 & 0.5 \\
\hline
\end{tabular}

$\% \mathrm{TBV}=$ percent of total brain volume

Volumes are presented as mean \pm S.D 